\title{
Non-Einsteinian black holes in generic 3D gravity theories
}

\author{
Metin Gürses* \\ Department of Mathematics, Faculty of Sciences, Bilkent University, 06800 Ankara, Turkey \\ Tahsin Çă̆rı Şişman (10 ${ }^{\dagger}$ \\ Department of Astronautical Engineering, University of Turkish Aeronautical Association, \\ 06790 Ankara, Turkey \\ Bayram Tekin $\oplus^{\ddagger}$ \\ Department of Physics, Middle East Technical University, 06800 Ankara, Turkey
}

(Received 10 July 2019; published 25 September 2019)

\begin{abstract}
The Bañados-Teitelboim-Zanelli (BTZ) black hole metric solves the three-dimensional Einstein's theory with a negative cosmological constant as well as all the generic higher derivative gravity theories based on the metric; as such it is a universal solution. Here, we find, in all generic higher derivative gravity theories, new universal non-Einsteinian solutions obtained as Kerr-Schild type deformations of the BTZ black hole. Among these, the deformed nonextremal BTZ black hole loses its event horizon while the deformed extremal one remains intact as a black hole in any generic gravity theory.
\end{abstract}

DOI: 10.1103/PhysRevD.100.064053

\section{INTRODUCTION}

The black hole in $2+1$ dimensions, the BañadosTeitelboim-Zanelli (BTZ) metric [1,2], as a solution to vacuum Einstein's gravity with a negative cosmological constant, shares many of the features of the $(3+1)$ dimensional realistic Kerr black hole. Due to the local triviality of Einstein's gravity in $2+1$ dimensions, the BTZ solution has been a remarkable tool in exploring the quantum nature of the black hole geometry such as a microscopic description of black hole entropy (see the review [3] and the references therein). Three important features of the BTZ geometry should be stressed. First, being a locally Einstein metric, it solves all the metric based higher curvature gravity equations derived from the most general action

$$
I=\int d^{3} x \sqrt{-g} \mathcal{L}(\text { Riem, } \nabla \text { Riem, } \cdots)
$$

Such metrics are called universal which are unaffected by the quantum effects [4,5]. Generically, for dimensions

\footnotetext{
*gurses@fen.bilkent.edu.tr

tahsin.c.sisman@gmail.com

btekin@metu.edu.tr
}

Published by the American Physical Society under the terms of the Creative Commons Attribution 4.0 International license. Further distribution of this work must maintain attribution to the author(s) and the published article's title, journal citation, and DOI. Funded by SCOAP ${ }^{3}$. greater than three, Einstein metrics fail to solve higher derivative theories but in three dimensions since the Riemann tensor can be written in terms of the Einstein tensor $G_{\mu \nu}$ as $R_{\mu \alpha \nu \beta}=\epsilon_{\mu \alpha \sigma} \epsilon_{\nu \beta \sigma} G^{\sigma \rho}$, any Einsteinian solution also solves the higher derivative theory as long as the cosmological constant is tuned accordingly. This fact is quite important and paves way to study the Einstein metrics such as the BTZ black hole as solutions to the low energy quantum theory of gravity at any scale defined by the action (1) where the nonmetric fields are set to zero or constant values. Secondly, the BTZ geometry can be dressed with two arbitrary functions to represent all the locally Einsteinian metrics yielding the Bañados geometry as [6]

$$
\begin{aligned}
d s^{2}= & \ell^{2}\left[\frac{\mathrm{d} r^{2}}{r^{2}}+\left(r \mathrm{~d} u+\frac{1}{r} f(v) \mathrm{d} v\right)\right. \\
& \left.\times\left(r \mathrm{~d} v+\frac{1}{r} g(u) \mathrm{d} u\right)\right],
\end{aligned}
$$

where $u$ and $v$ are null coordinates. The geometry corresponds to the nonextremal rotating BTZ black hole for constant nonvanishing values of $f$ and $g$; and to the extremal rotating BTZ black hole when one of these constants becomes zero. Thirdly, within the cosmological Einstein's theory, the BTZ black hole has the uniqueness property under the conditions described in $[7,8]$.

Due to the importance of the BTZ black hole, one would like to know its uniqueness and also whether it is preserved as a black hole under the deformations described as $g_{\mu \nu}=\bar{g}_{\mu \nu}+h_{\mu \nu}$ in the generic higher derivative theory (1). 
Here, $h_{\mu \nu}$ is not a small perturbation, hence just like the BTZ black hole $\bar{g}_{\mu \nu}$, the deformed metric $g_{\mu \nu}$ is expected to solve the full field equations with the condition that the black hole property is kept intact. Without a further specification of the field equations of the theory, one cannot proceed further with this most general deformation in a theory independent way. Therefore, to keep the universal nature of the BTZ black hole under this deformation in the setting of the most general higher derivative theory, we shall consider a specific deformation which is called the Kerr-Schild-Kundt (KSK) type whose universality (i.e., it solves the generic gravity theory once a linear scalar partial differential equation is solved) has been shown in [9-11]. The KSK metric is in the form

$$
g_{\mu \nu}=\bar{g}_{\mu \nu}+2 V \lambda_{\mu} \lambda_{\nu}
$$

where $V$ is a scalar field and $\lambda$ is a null vector field which satisfy the properties

$$
\begin{array}{ll}
\lambda^{\mu} \lambda_{\mu}=0, & \nabla_{\mu} \lambda_{\nu} \equiv \xi_{(\mu} \lambda_{\nu)}, \\
\xi_{\mu} \lambda^{\mu}=0, & \lambda^{\mu} \partial_{\mu} V=0,
\end{array}
$$

for both the background and the full metric. The $\xi$ vector is defined via the second equation in (4) once the $\lambda$ null vector is chosen (a way to generate viable $\lambda$ vectors from smooth curves was given in [12]). For the KSK metrics, the Ricci tensor becomes

$$
R_{\mu \nu}=(\mathcal{Q} V) \lambda_{\mu} \lambda_{\nu}-\frac{2}{\ell^{2}} g_{\mu \nu},
$$

where $\ell$ is the AdS length and the operator $\mathcal{Q}$ is defined as

$$
\mathcal{Q} V \equiv\left(\bar{g}^{\mu \nu} \bar{\nabla}_{\mu} \bar{\nabla}_{\nu}+2 \xi^{\mu} \partial_{\mu}+\frac{1}{2} \xi^{\mu} \xi_{\mu}-\frac{2}{\ell^{2}}\right) V .
$$

Then, for the pure cosmological Einstein theory, the nonlinear field equations $R_{\mu \nu}=2 \Lambda g_{\mu \nu}$ become linear in $V$ and boil down to [13]

$$
\mathcal{Q} V=0,
$$

once the trace of the field equations is solved as $\Lambda=-1 / \ell^{2}$. Given the background metric in some local coordinates, one can find the local solution. For a general gravity theory with the highest derivative order of $(2 N+2)$ in the field equations with $N \geq 0$, the field equations reduce to $[9,10,14]$

$$
\prod_{n=1}^{N}\left(\mathcal{Q}-m_{n}^{2}\right) \mathcal{Q} V=0
$$

whose generic solution is $V=V_{E}+\sum_{n=1}^{N} V_{n}$ where the Einsteinian part $\left(V_{E}\right)$ and the other (massive) parts, assuming nondegeneracy, satisfy the following equations:

$$
\mathcal{Q} V_{E}=0, \quad\left(\mathcal{Q}-m_{n}^{2}\right) V_{n}=0 .
$$

One can also interpret these equations as transversetraceless perturbations of the background space; therefore, they correspond to massless and massive gravitons. In three dimensional Einstein's theory, since there are no gravitons, $V_{E}$ corresponds to pure gauge transformations when the deformation $h_{\mu \nu}$ is assumed to be a perturbation about the exact background. On the other hand, the $V_{n}$ solutions are the non-Einsteinian solutions with the Ricci tensor $R_{\mu \nu}=\left(\sum_{n=1}^{N} m_{n}^{2} V_{n}\right) \lambda_{\mu} \lambda_{\nu}-2 / \ell^{2} g_{\mu \nu}$.

\section{DEFORMATIONS OF BTZ}

Along the lines described above, let us consider the deformations of the BTZ black hole

$$
d \bar{s}^{2}=-h \mathrm{~d} t^{2}+\frac{\mathrm{d} r^{2}}{h}+r^{2}\left(\mathrm{~d} \phi-\frac{j}{2 r^{2}} \mathrm{~d} t\right)^{2},
$$

with $h(r)=-m+\frac{r^{2}}{\ell^{2}}+\frac{j^{2}}{4 r^{2}}$. We shall call the generic deformation BTZ waves since the general solution will be of the wave form depending on the null coordinates. As we shall show below, among these only a subclass will remain a black hole. In (8), $m$ and $j$ are constants representing the mass and angular momentum, respectively. The outer and inner horizons of the black hole are located at

$$
r_{ \pm}^{2}=\frac{m \ell^{2}}{2}\left(1 \pm \sqrt{1-\frac{j^{2}}{m^{2} \ell^{2}}}\right)
$$

which coalesce for the extremal case $j= \pm m \ell$ at $r_{0}^{2}=m \ell^{2} / 2$.

To understand if and how the black hole nature of the BTZ metric is changed by the KSK deformation, let us study the event horizon. In the generic case, the symmetries of the BTZ geometry are no longer symmetries of the KSK geometry. Hence, the detection of the event horizon cannot be done with the Killing vectors; instead, since the horizons will be null hypersurfaces defined as level sets of $r$, let us consider where the surface normal $\partial_{\mu} r$ becomes a null vector in the BTZ-wave geometry as

$$
\Omega \equiv g^{\mu \nu} \partial_{\mu} r \partial_{\nu} r=0 .
$$

Using (3) and (8), $\Omega$ becomes

$$
\begin{aligned}
\Omega & =h(r)-2 V\left(\lambda^{\mu} \partial_{\mu} r\right)^{2} \\
& =2 V\left(t, r_{ \pm}, \phi\right)\left(\left.\lambda^{r}\right|_{r=r_{ \pm}}\right)^{2} .
\end{aligned}
$$

Here, to have $\Omega=0, V\left(t, r_{ \pm}, \phi\right)=0$ is a possibility but recall that the metric function $V$ must satisfy a theory dependent differential equation. Then, to keep the BTZ black hole intact in a theory independent way, 


$$
\left.\lambda^{r}\right|_{r=r_{ \pm}}=0
$$

must be satisfied. In this way, one has the deformed black hole solutions for all generic gravity theories. There can be other black hole solutions where the profile function $V$ satisfies the condition $V\left(t, r_{ \pm}, \phi\right)=0$ or $h(r)-$ $2 V\left(\lambda^{\mu} \partial_{\mu} r\right)^{2}=0$ for different $r$ values. In these cases, since $V$ takes different functional forms in different gravity theories then such black hole solutions will be theory dependent; and given the theory, one can construct these.

Since we are interested in the KSK-type deformations of the BTZ black hole which keep the event horizon intact, we considered a null hypersurface of constant $r$ to locate the event horizon. However, for the KSK metric (3), in general, a null hypersurface of the form $F(t, r, \theta)=$ constant should be considered to locate a horizon as

$$
\bar{g}^{\mu \nu} \partial_{\mu} F \partial_{\nu} F-2 V(t, r, \theta)\left(\lambda^{\mu} \partial_{\mu} F\right)^{2}=0 .
$$

In addition to the undeformed event horizon given with (12), to have a deformed horizon for the KSK geometry, this equation should be considered which will be studied elsewhere [15]. The analysis of (13) in its full generality is a tedious task; however, to get some understanding, for the $\lambda_{\mu}=\partial_{\mu} u$ case, ${ }^{1}$ let us consider the $r=f(u)$ hypersurface which becomes null if

$$
0=h(r)-2 \lambda^{r}\left(\frac{d f}{d u}+V(t, r, \theta) \lambda^{r}\right)
$$

To have an equation in $r$ and $u$ with a solution $r=f(u)$, one must have $V=V(u, r)$ with a $\lambda^{r}$ depending only $r$. Then, the KSK property $\lambda^{\mu} \partial_{\mu} V=0$ reduces to

$$
\lambda^{r} \frac{\partial V}{\partial r}=0
$$

which requires either $\lambda^{r}=0$ or $V=V(u)$. For $\lambda^{r}=0$, (14) becomes $h(r)=0$ so it does not provide a generalization as $r=f(u)$. Thus, one needs to have $V=V(u)$ in general. For this case, $\partial_{\mu} V=V_{u} \lambda_{\mu}$ and $\bar{g}^{\mu \nu} \bar{\nabla}_{\mu} \bar{\nabla}_{\nu} V=0$, so $\mathcal{Q} V$ reduces

$$
\mathcal{Q} V=\left(\frac{1}{2} \xi^{\mu} \xi_{\mu}-\frac{2}{\ell^{2}}\right) V
$$

To obtain an Einsteinian solution, $\mathcal{Q} V=0$ must be satisfied which is the case for any $V=V(u)$ if $\xi^{2}=4 / \ell^{2}$. The condition $\xi^{2}=4 / \ell^{2}$ is satisfied for the BTZ waves constructed in the next section. Thus, one may find a solution for (14) indicating a null hypersurface of the form $r=f(u)$ exists if $V=V(u)$. However, the $V=V(u)$ solution is an

\footnotetext{
${ }^{1}$ This choice is motivated at the beginning of the next section.
}

Einsteinian metric which is already represented in the Bañados geometry. Note that this case also covers the shifted horizons, that is $r=$ constant but $r \neq r_{ \pm}$, by having $f=$ constant and $V=$ constant. On the other hand, to have a non-Einsteinian KSK geometry for an horizon of the form $r=f(u)$, the metric function $V=V(u)$ must satisfy

$$
\left(\mathcal{Q}-m_{n}^{2}\right) V_{n}=\left(\frac{1}{2} \xi^{\mu} \xi_{\mu}-\frac{2}{\ell^{2}}-m_{n}^{2}\right) V=0,
$$

where $m_{n}$ depends on the parameters of the higher derivative theory. Since $\xi^{\mu}$ is theory independent, (17) cannot be satisfied in general. Therefore, it is not possible to obtain a non-Einsteinian KSK geometry that has a horizon of the form $r=f(u)$. In [15], we will study more general horizon forms such as $r=f(u, \psi)$ with $\xi_{\mu}=\partial_{\mu} \psi$ which require more general $V$ beyond $V=V(u)$.

In the discussion below, we will show that the condition (12), which keeps the BTZ event horizon intact, can be satisfied if and only if the BTZ seed is extremal so that a subclass of the BTZ waves will be a deformed version of the extremal BTZ black hole.

\section{BTZ-WAVE CONSTRUCTION}

Now, let us obtain the BTZ-wave metrics by a direct construction. As a consequence of the second property in (4), let us choose the null one-form field $\lambda_{\mu}$ to be exact, $\lambda_{\mu}=\partial_{\mu} u(t, r, \phi)$. Then, the condition that $\lambda_{\mu}$ be null yields

$$
\begin{aligned}
& -\left(\frac{\partial u}{\partial t}\right)^{2}-\frac{j}{r^{2}} \frac{\partial u}{\partial t} \frac{\partial u}{\partial \phi}+\left(\frac{h}{r^{2}}-\frac{j^{2}}{4 r^{4}}\right)\left(\frac{\partial u}{\partial \phi}\right)^{2}+h^{2}\left(\frac{\partial u}{\partial r}\right)^{2} \\
& =0
\end{aligned}
$$

Notice that all coefficients are a function of $r$, so the easiest way to satisfy the nullity condition is to consider a $u$ whose derivatives are either a function of $r$ or a constant $\mathrm{as}^{2}$

$$
u(t, r, \phi)=c_{1} t+c_{2} \phi+w(r) .
$$

This ansatz provides a solvable set of differential equations for the KSK metric properties. The solution can be put in a simpler form if the BTZ metric is written in terms of $r_{ \pm}$ with $h(r)=\frac{\left(r^{2}-r_{+}^{2}\right)\left(r^{2}-r_{-}^{2}\right)}{r^{2} \ell^{2}}$ and $j=\frac{2 \sigma r_{+} r_{-}}{\ell}$ where $\sigma$ represents the direction of rotation which we choose to be $\sigma=+1$. For this nonextremal BTZ seed, the $\lambda_{\mu}$ and $\xi_{\mu}$ one-forms are found to be

$$
\lambda_{\mu}=\left(1, \frac{\ell^{2} r\left(r_{+}+\epsilon r_{-}\right)}{\left(r^{2}-r_{+}^{2}\right)\left(r^{2}-r_{-}^{2}\right)}, \epsilon \ell\right)
$$

\footnotetext{
${ }^{2}$ There can be other choices for the function $u$ providing different solutions which will be discussed elsewhere [15].
} 
and

$$
\xi_{\mu}=\left(-\frac{r_{+}+\epsilon r_{-}}{\ell^{2}},-\frac{r(\alpha+\beta)}{\ell^{2} \alpha \beta}, \frac{\epsilon r_{+}+r_{-}}{\ell}\right),
$$

where $\epsilon$ is equal to $\pm 1, \alpha$ and $\beta$ are defined as $\alpha(r)=$ $\left(r^{2}-r_{+}^{2}\right) / \ell^{2}$ and $\beta(r)=\left(r^{2}-r_{-}^{2}\right) / \ell^{2}$. From (20), $\lambda^{r}$ can be calculated to be

$$
\lambda^{r}=h(r) \lambda_{r}=\frac{r_{+}+\epsilon r_{-}}{r} .
$$

The black hole event horizon condition (12) is not satisfied, so the BTZ deformation for the nonextremal case is not a black hole in the generic theory. Yet, the resulting metric is a solution to the generic theory if $V$ satisfies the constraint $\lambda^{\mu} \partial_{\mu} V=0$ and (6) for the specific theory. The constraint can be solved in a theory independent way and the solution is

$$
\begin{aligned}
V(t, r, \phi)= & \mathcal{F}\left(t+\frac{r_{+} \ln \alpha-\epsilon r_{-} \ln \beta}{2(\beta-\alpha)},\right. \\
& \left.\phi+\frac{r_{-} \ln \alpha-\epsilon r_{+} \ln \beta}{2(\beta-\alpha)}\right),
\end{aligned}
$$

where $\mathcal{F}$ is a smooth function.

Above, we discussed the nonextremal case, now let us focus to the extremal case $j=m \ell$ with $h(r)=\frac{\left(r^{2}-r_{0}^{2}\right)^{2}}{\ell^{2} r^{2}}$ and $j=\frac{2 r_{0}^{2}}{\ell}$. For this case, the sign choice $\epsilon$ becomes important as one arrives at two different metrics. For $\epsilon=+1$, with a similar construction as in the nonextremal case, the $\lambda_{\mu}$ and $\xi_{\mu}$ one-forms become

$$
\lambda_{\mu}=\left(1, \frac{2 r r_{0} \ell^{2}}{\left(r^{2}-r_{0}^{2}\right)^{2}}, \ell\right)
$$

and

$$
\xi_{\mu}=\left(-\frac{2 r_{0}}{\ell^{2}},-\frac{2 r}{r^{2}-r_{0}^{2}}, \frac{2 r_{0}}{\ell}\right)
$$

From (23), $\lambda^{r}$ can be calculated to be

$$
\lambda^{r}=\frac{2 r_{0}}{r} .
$$

Again, the black hole event horizon condition (12) is not satisfied, so the BTZ deformation for the extremal case with $\epsilon=+1$ is not a black hole in the generic theory.

For $\epsilon=-1$, the KSK metric construction for the extremal case differs in a subtle way from the nonextremal construction such that (18) requires $w(r)$ in (19) to be constant. As a result, the $\lambda_{\mu}$ and $\xi_{\mu}$ one-forms become

$$
\lambda_{\mu}=(1,0,-\ell),
$$

and

$$
\xi_{\mu}=\left(0, \frac{2 r}{r_{0}^{2}-r^{2}}, 0\right)
$$

From (25), $\lambda^{r}$ can simply be found to be

$$
\lambda^{r}=0
$$

This time, the black hole event horizon condition (12) is satisfied, so the BTZ deformation for the extremal case with $\epsilon=-1$ is a black hole in the generic theory. Here, the metric function $V$ must satisfy $\lambda^{\mu} \partial_{\mu} V=0$ yielding

$$
\frac{\ell^{2}}{r^{2}-r_{0}^{2}}\left(\ell \frac{\partial V}{\partial t}+\frac{\partial V}{\partial \phi}\right)=0,
$$

with the solution

$$
V=V(t-\ell \phi, r) .
$$

The explicit form of $V$ will be given below for Einstein's theory and the new massive gravity (NMG) [16].

\section{A. Extremal-BTZ wave solution of Einstein's gravity}

We showed that the only possible KSK deformation of a BTZ black hole which keeps the black hole nature intact is the extremal BTZ black hole deformed with the constant null vector field of $\lambda_{\mu}=(1,0,-\ell)$. Now, let us find the metric function $V$ for the cosmological Einstein's gravity by solving (5). With (26), the field equation for $V$ becomes

$$
r \frac{\partial^{2}}{\partial r^{2}} V_{E}(u, r)-\frac{\partial}{\partial r} V_{E}(u, r)=0,
$$

where we defined $u=t-\ell \phi$ which is in fact the generating function for $\lambda_{\mu}$ as $\lambda_{\mu}=\partial_{\mu} u$. If $r \neq r_{0}$, the Einsteinian solution becomes

$$
V_{E}(u, r)=c_{1}(u) r^{2}+c_{2}(u),
$$

yielding the metric

$$
d s^{2}=d \bar{s}^{2}+2\left(c_{1}(u) r^{2}+c_{2}(u)\right)(\mathrm{d} t-\ell \mathrm{d} \phi)^{2},
$$

where $d \bar{s}^{2}$ is the extremal BTZ seed. This result is consistent with the Bañados geometry (2) and the analysis of [8]. As in the case of the Bañados geometry which dresses the BTZ black hole with two arbitrary functions, our generic solution with arbitrary $c_{1}(u)$ and $c_{2}(u)$ are of the nonlinear wave type which we called the BTZ wave. To understand this solution better, we can compute its mass and angular momentum using the Abbott-Deser approach [17]. Assuming $c_{1}(t-\ell \phi)=c_{2}(t-\ell \phi)=0$ and $r_{0}=0$ to be the background, the mass corresponding to the background timelike Killing vector $\zeta^{\mu}=(-1,0,0)$ is 
$M=m+\frac{2}{\pi} \int_{0}^{2 \pi} d \phi c_{2}(t-\ell \phi)$, and the angular momentum corresponding to the background Killing vector $\zeta^{\mu}=$ $(0,0,1)$ is $J=m \ell+\frac{2 \ell}{\pi} \int_{0}^{2 \pi} d \phi c_{2}(t-\ell \phi)$. We have kept mass and angular momentum computation with generic $c_{1}(u)$ and $c_{2}(u)$. Since this solution is no longer stationary, its mass angular momentum are time dependent via these functions. Note that the extremality condition is intact as $J=M \ell$. The function $c_{1}(t-\ell \phi)$ corresponds to a pure gauge and does not appear in the mass and angular momentum expressions. Of course, for a stationary black hole solution, the arbitrary $u$ dependent functions should be taken as constants as we mentioned for (2). Then, one obtains time-independent mass and angular momentum. The discussion is exactly like the case of the Bañados metric $[6,8]$.

\section{B. Extremal-BTZ wave solution of NMG}

Now, we study the solution of cosmological new massive gravity given with the action

$$
I=-\frac{1}{\kappa^{2}} \int d^{3} x \sqrt{-g}\left(R-2 \Lambda_{0}+L^{2} K\right),
$$

whose field equations are

$$
G_{\mu \nu}+\Lambda_{0} g_{\mu \nu}-\frac{L^{2}}{2} K_{\mu \nu}=0,
$$

where $K_{\mu \nu}=2 \square R_{\mu \nu}-\frac{1}{2}\left(\nabla_{\mu} \nabla_{\mu}+g_{\mu \nu} \square\right) R+4 R_{\mu \alpha \nu \beta} R^{\alpha \beta}-$ $\frac{3}{2} R R_{\mu \nu}-g_{\mu \nu} K$ and the trace $K=g^{\mu \nu} K_{\mu \nu}=R_{\mu \nu} R^{\mu \nu}-\frac{3}{8} R^{2}$. Putting the metric of the extremal BTZ wave defined by $\lambda_{\mu}$ given in (25) yields the field equations

$$
\begin{gathered}
\frac{1}{\ell^{2}}+\Lambda_{0}+\frac{L^{2}}{4 \ell^{4}}=0, \\
\left(\mathcal{Q}-m_{g}^{2}\right) \mathcal{Q} V=0,
\end{gathered}
$$

where $m_{g}^{2}$ is the mass of the spin-2 graviton of the NMG theory given as

$$
m_{g}^{2}=\frac{1}{L^{2}}-\frac{1}{2 \ell^{2}}
$$

The first equation determines the effective cosmological parameter $\ell$. The second equation (35) determines the metric function $V$ and has the general solution

$$
V(u, r)=V_{E}(u, r)+V_{p}(u, r),
$$

where $u=t-\ell \phi$ and $V_{E}$ is the Einsteinian solution (31) while $V_{p}$ is the solution of the massive operator $\left(\mathcal{Q}-m_{g}^{2}\right)$ which can be found as

$$
\begin{aligned}
V_{p}(u, r)= & c_{3}(u)\left(r^{2}-r_{0}^{2}\right)^{(1+p) / 2} \\
& +c_{4}(u)\left(r^{2}-r_{0}^{2}\right)^{(1-p) / 2},
\end{aligned}
$$

with $p \equiv \sqrt{m_{g}^{2} \ell^{2}+1}$. The reality of $p$ is equivalent to the Breitenlohner-Freedman (BF) bound [18]. It is important to note that the solution (37) to this quadratic theory solves all higher curvature theories as long as the corresponding effective cosmological constant equation is satisfied. Using the construction of [19], one can show that the finiteness of mass and angular momentum requires $c_{3}(u)=c_{4}(u)=0$ for $0<p<1, c_{3}(u)=0$ for $1<p$, or $c_{4}(u)=0$ for $p<-1$ yielding the mass $M=m\left(1+\frac{2}{2 p^{2}-1}\right)$ and the angular momentum $J=M \ell$ such that extremality is kept intact.

\section{CONCLUSIONS}

We have studied the exact deformation of the BTZ black hole in the context of generic gravity and showed that the nonextremal black hole loses its exact horizon and the resulting deformed metric is of wave type, which we called the BTZ wave. Surprisingly, the deformed extremal black hole remains a black hole. There are several ways to read this result: First, the nonextremal BTZ is unique in generic gravity while the extremal one is not as in the case of Einstein's theory; second, considering the deformations as generic quantum or classical corrections, the nonextremal BTZ is not preserved as a black hole solution to the generic gravity while the extremal one remains a black hole in any generic gravity theory. Lastly, regarding the $r=0$ singularity after the KSK deformation, note that all the curvature invariants of the KSK metrics are constant; therefore, there is no curvature singularity.
[1] M. Banados, C. Teitelboim, and J. Zanelli, The Black Hole in Three-Dimensional Space-Time, Phys. Rev. Lett. 69, 1849 (1992).

[2] M. Banados, M. Henneaux, C. Teitelboim, and J. Zanelli, Geometry of the $(2+1)$ black hole, Phys. Rev. D 48, 1506 (1993); Erratum, Phys. Rev. D 88, 069902(E) (2013).
[3] S. Carlip, Black hole thermodynamics, Int. J. Mod. Phys. D 23, 1430023 (2014).

[4] G. W. Gibbons, Quantized fields propogating in plane waves spacetimes, Commun. Math. Phys. 45, 191 (1975).

[5] A. A. Coley, G. W. Gibbons, S. Hervik, and C. N. Pope, Metrics with vanishing quantum corrections, Classical Quantum Gravity 25, 145017 (2008). 
[6] M. Banados, Three-dimensional quantum geometry and black holes, AIP Conf. Proc. 484, 147 (1999).

[7] M. Rooman and P. Spindel, Uniqueness of the asymptotic AdS(3) geometry, Classical Quantum Gravity 18, 2117 (2001).

[8] C. Li and J. Lucietti, Three-dimensional black holes and descendants, Phys. Lett. B 738, 48 (2014).

[9] M. Gurses, S. Hervik, T. C. Sisman, and B. Tekin, Antide Sitter-Wave Solutions of Higher Derivative Theories, Phys. Rev. Lett. 111, 101101 (2013).

[10] M. Gurses, T. C. Sisman, and B. Tekin, AdS-plane wave and pp-wave solutions of generic gravity theories, Phys. Rev. D 90, 124005 (2014).

[11] M. Gurses, T. C. Sisman, and B. Tekin, Kerr-Schild-Kundt metrics are universal, Classical Quantum Gravity 34, 075003 (2017).

[12] M. Gurses, T. C. Sisman, and B. Tekin, From smooth curves to universal metrics, Phys. Rev. D 94, 044042 (2016).
[13] M. Gurses, T. C. Sisman, and B. Tekin, New exact solutions of quadratic curvature gravity, Phys. Rev. D 86, 024009 (2012).

[14] M. Gurses, T. C. Sisman, and B. Tekin, Gravity waves in three dimensions, Phys. Rev. D 92, 084016 (2015).

[15] M. Gurses, T. C. Sisman, and B. Tekin (to be published).

[16] E. A. Bergshoeff, O. Hohm, and P. K. Townsend, Massive Gravity in Three Dimensions, Phys. Rev. Lett. 102, 201301 (2009).

[17] L.F. Abbott and S. Deser, Stability of gravity with a cosmological constant, Nucl. Phys. B195, 76 (1982).

[18] P. Breitenlohner and D.Z. Freedman, Positive energy in anti-De Sitter backgrounds and gauged extended supergravity, Phys. Lett. 115B, 197 (1982).

[19] S. Deser and B. Tekin, Gravitational Energy in Quadratic Curvature Gravities, Phys. Rev. Lett. 89, 101101 (2002). 